Colonisation, développement et sciences sociales. Éléments pour une sociologie de la constitution du champ des "arts et sciences du développement" dans les sciences sociales francophones belges

\title{
Marc Poncelet
}

\section{OpenEdition}

Journals

Édition électronique

URL : http://journals.openedition.org/apad/2503

DOI : $10.4000 /$ apad.2503

ISSN : 1950-6929

Éditeur

LIT Verlag

Édition imprimée

Date de publication : 1 décembre 1993

Référence électronique

Marc Poncelet, « Colonisation, développement et sciences sociales. Éléments pour une sociologie de la constitution du champ des "arts et sciences du développement" dans les sciences sociales

francophones belges ", Bulletin de l'APAD [En ligne], 6| 1993, mis en ligne le 10 mars 2008, consulté le 08 septembre 2020. URL : http://journals.openedition.org/apad/2503 ; DOI : https://doi.org/10.4000/ apad. 2503

Ce document a été généré automatiquement le 8 septembre 2020

Bulletin de I'APAD 


\title{
Colonisation, développement et
} sciences sociales. Éléments pour une sociologie de la constitution du champ des "arts et sciences du développement" dans les sciences sociales francophones belges

\author{
Marc Poncelet
}

1 En quatre décennies, le "développement" est passé du statut théorique de mouvement socio-historique à celui de mythe. Du coup, c'est un espace-clé de la production des sciences sociales qui semble réclamer une analyse plus fine en termes épistémologiques et sociologiques.

2 La notion de développement a synthétisé la plupart des composantes imaginaires et conceptuelles d'une mondialisation de la notion de progrès précédemment soumise à celles de Civilisation (mouvement civilisateur) et d'Evolution. Vu l'ampleur qu'elle a prise au XXe siècle et surtout après la seconde guerre mondiale pour ce qui concerne les aires non occidentales, il est important de noter que les sciences sociales y ont trouvé (et pour une bonne part, ont contribué à sa formation) un lieu inédit d'investissement et ont joué un rôle capital dans ce mouvement séculaire de recul (peut-être seulement apparent) des garants métaphysiques au profit d'un volontarisme prométhéen fondé sur l'entendement scientifique. Le développement, malgré ou grâce à sa constante indéfinition, a ainsi été la pierre de touche de la mondialisation d'un corpus d'idées de formation pourtant récentes, d'un ethos inédit du savoir et du savant et surtout d'un credo. Ceci étant particulièrement caractéristique des jeunes sciences sociales qui avancèrent sur ce terrain a mains nues.

3 La renaissance des sciences sociales au lendemain de la seconde guerre tient à plusieurs facteurs. Parmi ceux-ci, on fait trop souvent l'impasse sur la "découverte" de la question coloniale telle qu'elle est révélée entre et 1950. La politique sociale visant l'évolution et le progrès du monde indigène fournit un espace ou s'engouffre le credo social-scientiste. 
Dans le cas du Congo belge, face à la prolétarisation de masse qui témoigne d'une puissance exceptionnelle de transformation du milieu, c'est la politique indigène qui va constituer tardivement le lieu d'un réformisme confus et hésitant. Mais pour ce qui nous concerne, ce sera aussi la matrice et le lieu d'investissement original des "social scientists" métropolitains, l'occasion d'une renaissance de "l'ethnologie techné" (Leclerc, 1979) et de la formation d'une utopie technocratique d'ingénierie sociale. Le grand capital colonial, notamment minier, est alors confronté à l'obsolescence de son système ultra-paternaliste de gestion de la main-d'oeuvre et aux effets pervers de ses premières tentatives de stabilisation de celle-ci.

$5 \quad$ C'est donc le moment où la mobilisation forcée du travail agricole et l'expropriation systématique atteint ses limites. Le "bien-être et l'éducation des indigènes" - thèmes nouveaux - quittent le ressort de la bienfaisance et de l'évangélisation. Une utopie technocratique se meut autour du credo de la guidance scientifiquement contrôlée du progrès social.

6 Le Développement est alors "bricolé" par des sciences sociales comme alternative à la Civilisation. Le savant prend le pas sur le missionnaire. Le Congo devient précipitamment indépendant. C'est dans ce contexte que les infrastructures socio-scientifiques des arts et sciences du développement sont mises en place pour les deux décennies suivantes durant lesquelles se constituent sur ces terrains, de nouveaux marchés savants.

7 Autour du "développement", divers débats se sont noués depuis la fin des années 1970.

8 La critique théorique, méthodologique, épistémologique et finalement culturelle du "développement" lui-même a succédé à une critique de la domination post-coloniale inspirée de formalisations qui restaient largement de nature économique. Le mouvement de démarxification des sciences sociales, les lectures culturelles de la crise qui s'ouvre avec la décennie 1970, l'héritage des mouvements occidentaux de contestations culturelles, les avatars des "other developments", la professionnalisation croissante du champ et enfin l'effondrement du référent imaginaire du tiers-mondisme débouchent ainsi sur plusieurs mouvements de réflexion.

9 Le plus connu est l'expression d'un pessimisme radical quant au "développement" relu comme cheval de Troie de l'occidentalisation destructrice et stérile. Il s'alimente d'une redécouverte des cultures et des relations inter-culturelles relues et reconstruites selon les clés culturalistes. On pense, pour la littérature française à $\mathrm{S}$. Latouche, mais il s'agit aussi d'un genre d'école internationale de l'anti-développementalisme radical. Elle semble assumer actuellement la fonction prophétique dans le champ des "savoir-développer" et, si elle entretient quelques parentés ou solidarité théorique avec quelques prestataires de services thérapeutiques immédiats et de petites officines de guérison (ONG), c'est pour les enjoindre d'abandonner illico toute velléité d'intervention car le constat est sans recours : "it did not work" ${ }^{1}$ (W. Sachs, 1992).

10 Plus discrètement, c'est un processus de relecture de l'histoire coloniale et des rapports métropoles-colonies qui a alimenté une bonne littérature où historiens et anthropologues ont redoublé d'efforts pour nous expliquer que "tout cela était bien plus compliqué" que ne l'avaient laissé entendre les théories diversement dépendantistes. Bref, là aussi, la complexité retrouvait ses droits à l'instar du local, des dynamiques du dedans et de l'historicité. Simultanément, on mesurait ici comme dans toute la sociologie, le retour de l'acteur. (Piault, 1987, Marseille, 1984, Boiral, Lantéri, Olivier de Sardan, 1985).

11 Un troisième mouvement de la réflexion, un troisième chantier prend forme qui rencontre enfin de front une question le plus souvent éludée, y compris par les 
polémiques les plus radicales. Il ne s'agit plus de tester ou contester des théories, de dénoncer des idéologies, des perversions, des manipulations. Il s'agit d'examiner, en faisant retour sur soi et ses outils, le rôle et les fonctions des sciences sociales dans la constitution et l'institutionnalisation des "savoir-développer" concourant à la fabrication d'un paradigme, ou à tout le moins d'un espace du savoir, qui semble avoir sous-tendu l'objectivation et la mondialisation d'un formidable complexe idéologique. Avec le "développement", les sciences sociales et humaines en particulier ont semblé trouver un espace d'investissement inégalé à ce jour. A travers ce méta-paradigme, elles eurent l'opportunité de renouer avec une vocation "comtienne", elles trouvèrent la clé de leur universalisation jusqu'ici très incomplète. Comment le fabriquèrent-elles?

Dans l'histoire de la production et de la diffusion des idées, les sciences sociales ont-elles été davantage associées à la genèse et à la dynamique d'un tel produit ?

Dans cette perspective, l'actualité éditoriale nous offre déjà de nombreux éléments d'un regard qu'on peut qualifier d'épistémologique. Diverses publications tendent en effet à proposer une lecture rétrospective de la formation des théories, de la création des objets "sous-développement" et "développement" et des principaux credo qui ont jalonné la trajectoire des "savoir-développer". Je pense ici par exemple à la publication patronnée par la Banque Mondiale et dirigée par G. Meire (1988) intitulée Les pionniers du développement, à l'article de P. Hugon (1990) consacré à l'évolution d'une économie française du développement, à l'ouvrage de H. W. Arndt Development, history of an idea... qui ont tous une vocation disciplinaire (ici l'économie). Du côté des sociologues, les essais se multiplient pour situer également leur discipline dans le processus collectif de fabrication du "développement". Voir entre autres la revue Tiers-Monde, $\mathrm{N}^{\circ} 90,1982$ et $\mathrm{N}^{\circ} 100,1984$; Boudon (1984); les publications de Goussault (1987); Gousseault et Guichaoua (1989); les contributions de Guichaoua, Touraine et Boudon au volume de L'Année Sociologique 1992 et les publications en langue anglaise: Harrison (1988); Dube (1988); Webster (1990); Worsley (1984).

La plupart de ces travaux suivent trois axes - histoire (voire historiographie) épistémologie - sociologie de la connaissance. C'est ce dernier que je tente de privilégier dans un projet de recherche intitulé "invention et trajectoire de la notion de développement dans les sciences sociales".

Celui-ci renvoie à la logique des champs scientifiques et originellement, à leurs rapports aux faits coloniaux.

Dans le cadre des sciences sociales en Belgique, toute saisie du champ scientifique constitué autour des "arts et sciences du développement" entendu comme le lieu général d'énonciation et de formation d'enjeux relatifs au changement social hors d'Europe, renvoie à l'origine des sciences coloniales.

17 Celles-ci connaissent une mutation au lendemain de la guerre avec la problématisation de la question sociale indigène principalement au Congo (Zaïre). C'est précisément ce moment qui traduit à mes yeux le premier mouvement de constitution et d'autonomisation d'un champ spécifiquement savant propre aux arts et sciences du développement. Le paysage actuel, universitaire et savant dans l'ensemble, en porte encore l'empreinte évidente. Ce que je considère, faute de mieux, comme les "infrastructures socio-scientifiques" y ont vu le jour et ont sous-tendu le travail théorique, idéologique et pratique qui fonde un champ des "savoir-développer". 
18 La curieuse aisance avec laquelle se renouvellent les productions savantes autour du "développement" induit en fait une perspective tronquée. En se faisant critiques par excellence (et de plus en plus nettement) des pratiques des "développeurs", elles occultent ce que le "développement" leur doit en propre tant à un niveau fondamentalement épistémologique qu'au niveau de leurs logiques sociales de fonctionnement ${ }^{2}$.

19 Je propose ici un aperçu et une esquisse d'un site qui correspond au moment explicitement développemental du processus colonial au Congo belge (Zaïre). Il illustre bien à mon sens à la fois la continuité et les ruptures de la reconversion de la Civilisation en Développement, jette une lumière crue sur les logiques savantes qui furent mises en œuvre et fournit un remarquable cas "d'alchimie" locale, voire d'invention de différents credo et pratiques "des savoir-développer".

20 Un petit détour par les sciences coloniales s'impose cependant En effet, le développement apparaît souvent comme la problématique post-coloniale par excellence. C'est évidemment historiquement faux. Mais il reste que le développement a fait office d'espace de décolonisation (réussie ou non) des sciences sociales. Le développement a "pris" (au sens du ciment qui prend) sur base d'une énorme amnésie qui a autorisé sa projection dans l'espace des théories politiquement "progressistes". Ce mouvement a entièrement escamoté la révolution épistémologique voire cognitive que supposait la décolonisation en matière d'analyse des sociétés en cause. Dans le cas belgo-congolais, il aura fallu passer d'un mode d'analyse entérinant de fait l'absolue nécessité de la tutelle à une entreprise supposant de conférer une quelconque potentialité aux acteurs congolais. Dans la pratique, celle de la coopération ou de la gestion des grandes entreprises par exemple, cette question ne se posait même pas dans la mesure où l'indépendance, aux yeux de tous, ne mettait pas en cause l'effectivité et la nécessité de la tutelle. Par contre, au niveau des sciences sociales, et alors que les infrastructures socio-scientifiques, épistémologiques et cognitives du développement seront celles du colonialisme tardif, la "non-révolution" de l'indépendance doit être interrogée comme l'un des moments clés de la formation du champ des arts et sciences du développement.

21 Je ne ferai pas référence à une autre source possible spécifiquement métropolitaine de l'invention savante du "développement", à savoir les recherches socio-historiques sur le développement industriel en Europe. Ce secteur dont les influences théoriques seront indéniables dans les années 1960 et 1970 restera sans rapport avec les marchés savants nés de la problématisation coloniale tardive sinon à leur marges critiques et académiques.

22 L'entreprise léopoldienne et les tractations internationales qui aboutissent à la reconnaissance de l'Etat Indépendant du Congo en 1885 (EIC) autorisent à avancer que le Congo, qui ne deviendra une colonie belge qu'en 1909 avec ce qu'il est convenu d'appeler la "reprise", sera avant tout une colonie du grand capital européen puis, de plus en plus, spécifiquement belge.

23 Léopold II dont "l'CEuvre" est contestée de manière récurrente au niveau international, a très vite saisi l'opportunité d'associer le monde savant à ses ambitions, non seulement parce que l'exploitation économique met à l'avant-plan les deux figures savantes que sont "l'agronome gendarme" et le "géologue conquérant", mais plus généralement parce que le discours scientifique global est une composante essentielle de la légitimité 
de l'entreprise : la mise en exploitation, la mise en valeur deviennent des challenges financiers mais aussi scientifiques.

Léopold II puis l'État belge créent ainsi des institutions savantes coloniales, en Belgique évidemment. Elles ont forme de clubs et sont indépendantes des universités. Elles fonctionnent par cooptation et réunissent les très minces "élites qui s'intéressent à l'Æuvre civilisatrice du Roi" dans un réseau original dominé par la haute finance et les deux autres corps de la trinité coloniale belge: l'Administration coloniale et les missions.

Entre autres institutions, l'Institut Royal Colonial Belge, l'Institut Royal International Colonial, le Musée d'Afrique Centrale, l'Institut des Parcs Nationaux, la Société d'Étude Coloniale... Le Congrès Colonial National Belge sera pour sa part l'œuvre d'Albert 1. Elles persistent aujourd'hui sous d'autres dénominations. L'enseignement colonial sera aussi du domaine réservé des autorités coloniales. Médecins et fonctionnaires passent impérativement par des institutions spécialisées qu'elles contrôlent. Ce n'est qu'au lendemain de la seconde guerre mondiale que les universités ouvrent des sections coloniales. L'Université de Liège par exemple, précise pourtant bien qu'elles ne sont pas destinées aux fonctionnaires ni aux médecins, mais aux candidats cadres du secteur privé. L'enseignement religieux a pour sa part ses propres structures de formation indépendantes de l'Etat belge. J. Vanderlinden ironise à juste titre sur la réalité de l'enseignement relatif à l'Afrique dans les universités jusqu'en 1960 (Vanderlinden, 1985).

26 Si on a coutume de dire, et à juste titre, que dès l'origine "l'œuuvre coloniale" ne fut jamais populaire en métropole, il convient de reconnaître qu'elle fut essentielle à la constitution du monde scientifique belge. Et celui-ci ne se montra pas ingrat! C. Young épinglera plus tard "l'absence d'une tradition d'études critiques de l'effort colonial dans les milieux universitaires".

Médecins, géologues, agronomes, juristes, administrateurs et missionnaires exerceront la fonction d'œil total du panoptique colonial belge au Congo, un dispositif "remarquable" selon le même auteur qui insiste sur "l'incroyable contrôle qu'il réussit à exercer sur presque tout l'éventail des activités humaines". L'élite coloniale se recrute de manière très contrôlée dans certaines couches traditionnellement dominantes en métropole. Après la seconde guerre mondiale, tout recrutement dans l'administration coloniale supposera un diplôme universitaire.

Les institutions scientifiques de Belgique sont étroitement associées aux affaires (et bien sûr aux bonnes affaires en premier lieu) coloniales et aux aventures impérialistes outre-mer de la haute finance emmenée par le Roi Souverain. Les biographies en témoignent. Qu'on en juge: Avant de publier son "Etude sur la situation de l'EIC," ouvrage qui annonce et plaide pour la reprise, F Cattier professeur de droit colonial à l'ULB (Université Libre de Bruxelles), aura été Secrétaire Général de la Compagnie d'Extrême Orient et de la Compagnie de Kaïping dont Thys, autre "héros colonial", est président administrateur et encore... conseiller juridique du Roi de Siam. Ce même Cattier qui participa à la rédaction de la charte coloniale fût évidemment membre du Conseil Colonial, collaborateur d'autres "immenses figures" coloniales: J Jadot et $\mathrm{E}$ Francqui ("pères" du Katanga). Avec ce dernier, ils jouèrent un rôle considérable dans la création de la Fondation Universitaire et du Fond National de la Recherche Scientifique dont Cattier sera d'ailleurs Président. Il sera aussi Administrateur Délégué et Vice-président de la Banque d'Outre Mer, puis Directeur, Vice-Gouverneur de la 
Société Générale de Belgique, Président de l'Union Minière du Haut Katanga (UMHK), fondateur de l'Agence Maritime Internationale...

Le Roi-Souverain ne ménagea pas non plus son énergie pour convaincre les élites intellectuelles contestant l'utilité de la colonisation au nom du libre échange CE. De Laveleye parmi d'autres).

L'espace colonial est en fait l'une des matrices d'un remarquable modèle de circulation des élites sociales et des éminences grises, modèle aussi de consolidation d'une élite savante nationale de facture récente et intimement associée au dense réseau de complexes cooptations qui renvoie à l'unionisme fondateur national.

31 Sur le terrain, les agronomes avec l'INEAC (Institut National d'Études Agronomiques au Congo, fleuron de l'agronomie tropicale principalement aux mains de l'administration coloniale et peuplée d'agronomes de l'Université de Louvain), disposeront dès les années 1930 d'un outil remarquable d'intervention toujours autoritaire et parfois techniquement catastrophique en milieu rural au service du travail obligatoire et des cultures d'exportation imposées sous diverses formes.

Si l'on se souvient des conditions de reconnaissance de l'EIC, de sa constitution tardive alors que l'argumentaire anti-colonial (libéral ou humanitaire) est déjà bien fourni, y compris en Belgique avec de grands noms des universités, on comprend que le Roi tenta de mobiliser les hommes de lettres et de sciences. Avec une remarquable constance, il réunit des savants de l'Europe mais aussi des diplomates de différents pays et parvint à se construire un espace de légitimité dans le concert des puissances coloniales. Il en va de même pour la Belgique-tutrice à qui l'€uvre échut. La valorisation scientifique du Congo ne fut jamais si grande que lorsque "l'œuuvre" était contestée. Ceci est une constante jusqu'à la décolonisation et ses instruments scientifiques lui survécurent... rebaptisés évidemment.

33 Léopold II est encore à l'origine de la première grande investigation sur le "matériau humain colonial". Avec les promoteurs de celle-ci, c'est à dire la première Société Belge de Sociologie et alors que "l'Æॄuvre" est contestée à l'étranger, une stratégie d'internationalisation de la recherche est astucieusement mise au point. Toutes les puissances coloniales et les Etats comptant des populations d'intérêt ethnologique sont associés.

Fondée en 1899, cette Société Belge de Sociologie est une société savante plutôt composée de catholiques. Elle prend donc corps indépendamment des initiatives de SOLVAY qui fonde à Bruxelles l'Institut Solvay des Sciences Sociales, ancêtre de l'Institut de Sociologie Solvay. Elle sera donc à l'origine de la première grande initiative menée en sciences sociales ayant pour objet les populations de L'EIC. Ce projet intitulé "Enquête sociologique sur les peuples de civilisation inférieure" est très vite élargi à "d'autres peuples incultes d'autrefois ou de race jaune" (de Bie, 1988 p. 114).

Missionnaires et agents de l'EIC seront les principaux collecteurs d'informations sur le terrain, mais un autre questionnaire est destiné à divers spécialistes chargés d'études monographiques et spécialisées. On demanda le concours de divers Etats intéressés et on est prêt à négocier des accords avec des sociétés étrangères. "On sent un grand enthousiasme, des plans grandioses, un souci d'organisation" (idem p. 114).

36 Le projet est effectivement d'une ampleur inouïe, il se développe promptement à l'initiative de Halkin, géographe liégeois et de Van Overbergh, Président de la Société et ami de Léopold II. 
37 La première démarche qui voit ses promoteurs établir des contacts dans divers pays européens et y recevoir un accueil enthousiaste débouche sur la constitution d'un Bureau International à l'occasion du Congrès International d'Expansion Économique Mondiale tenu à Mons en 1905. Lors de ce congrès, les éminents scientifiques présents plaident pour la création d'institutions scientifiques internationales spécifiquement coloniales et réclament une pédagogie de la colonisation qui doit être mise en œuvre en métropole. La Société Belge devient le Mouvement Sociologique International dont le Bureau élira domicile en Belgique. Chaque commission nationale aura en charge son ressort national et colonial. Un plan minutieux est proposé qui doit permettre la systématisation des résultats selon un schéma standard.

Pour ce qui concerne les monographies de populations de l'EIC, les publications seront nombreuses. Les livraisons du dorénavant "Mouvement" pour la période 1907-1910 comprennent quelques 4257 pages dont plus de la moitié est consacrée aux résultats de l'enquête ethnographique.

Cette enquête constitue la grande œuvre de la première Société Belge de Sociologie et vaudra à son président Van Overbergh la présidence du Bureau International d'Ethnographie.

Il s'agit là de la première percée d'une science sociale sur le terrain colonial belge. Elle dépasse par son objectif et son champ les travaux ethnographiques jusqu'ici exceptionnellement professionnels. Elle a mobilisé des fonctionnaires, des missionnaires, des agronomes, et autres observateurs patentés des mœurs indigènes qui avaient accès au terrain. Mais sa genèse indique que la colonie et son matériau humain est dorénavant objet spécifiquement savant. Elle a bien sûr fait l'objet de nombreuses critiques méthodologiques, mais elle s'inscrit parfaitement dans un dessin encyclopédique et savant où la sociologie au statut jusqu'ici incertain, entend rationaliser ses méthodes de collecte, de traitement et de présentation, et définir son champ. L'opération n'atteint cependant pas entièrement l'objectif scientifique qui consistait en l'appropriation centralisée du savoir ethnologique qui restera longtemps encore aux mains des" gens de terrain", principalement missionnaires ${ }^{3}$.

41 "L'CEuvre" sera bien, mais pour peu de temps, au centre d'un projet international consacrant l'universalité de l'objet savant colonial.

La campagne de la reprise (du Congo par l'État belge à titre de colonie) offrira une occasion de se manifester à un autre mouvement scientifique: le Mouvement Géographique Belge qui en deviendra le chef d'orchestre en reprenant à son compte les intérêts des grandes compagnies financières. Le Mouvement aura des publications régulières sur le Congo. Le discours de ces "géographes" va construire et donner une respectabilité académique à l'idée selon laquelle un abandon du Congo aurait des conséquences incalculables sur le développement scientifique belge et serait un péché mortel contre l'éthique du progrès scientifique.

43 Le rôle des sociétés de géographie dans la construction du champ colonial comme champ scientifique intimement dépendant des intérêts coloniaux économiques et politiques est largement attesté dans le cas de la France. De 1871 à 1881 onze nouvelles sociétés de ce type voient le jour. Au total elles regroupent quelques 10.000 membres (Girardet, 1972).

44 La relative discrétion sociologique internationale des années 1920 et 1930 n'est évidemment pas favorable au développement d'une sociologie coloniale. On note 
cependant un intérêt croissant de l'Institut Solvay (ULB). Louvain ne montre aucun investissement colonial particulier dans le domaine des sciences humaines hormis bien sûr ce qui touche aux missions.

Les juristes belges seront cependant à l'origine d'une impressionnante littérature qui se développera notamment dans le Bulletin des Juridictions indigènes et du Droit coutumier congolais où sont discutés les principaux thèmes de doctrine coloniale.

Les sciences naturelles appliquées (géologie, médecine, agronomie) et quelques sciences fondamentales accroissent considérablement leurs encyclopédies coloniales. Elles sont quelques fois mobilisées pour les grands travaux de l'administration coloniale et par les grandes entreprises. Cependant les grandes compagnies ont souvent leur personnel sur le terrain : ingénieurs, médecins, agronomes, etc. Ce sont les sociétés d'anciens étudiants qui joueront dès lors à partir du terrain un rôle capital de connexion et de structuration des positions savantes. Elles ont un principe institutionnel (une université, une grande école) et une vocation coloniale (exemple : les anciens de tel établissement au Katanga...) ou encore, plus corporatiste et régionale (les anciens ingénieurs issus de telle école en service à Léopoldville).

La valorisation scientifique passe toujours mais non plus exclusivement par les cercles savants spécifiquement coloniaux. Elle est positivement associée au progrès et à la mise en valeur.

L'ULB, établissement universitaire libre et professant le libre examen, entre en force dans le champ colonial dans les années 1920. Elle fournit de hauts cadres à l'administration. à la haute finance et développe une action propre par le biais d'un Centre pour la Recherche Médicale en Afrique Centrale (CEMUBAC) qui entend répondre aux initiatives louvanistes du même type (Fomulac à Kisantu).

La problématisation coloniale ou mise en valeur qui précède la problématisation du bien-être indigène, elle-même matrice du développement, franchit cependant un cap avec la crise des années 1930 qui est particulièrement grave au Congo, induisant des licenciements massifs et une récession révélatrice de la fragilité de cette économie de ponction.

50 Ainsi parmi les quelques initiatives universitaires, l'Institut Solvay (ULB) réunit un grands nombres de spécialistes de diverses disciplines, de coloniaux, d'universitaires et étudiants de toutes les universités, et de hauts responsables politiques et administratifs pour traiter de "l'extraordinaire développement de la colonie et de la crise qui la touche", alimentant à nouveau le scepticisme métropolitain. Il s'agit d'examiner «les problèmes théoriques que posent la colonisation, l'organisation de la nouvelle communauté sociale que nous assumons au Congo à travers les industries, l'agriculture, les transports, le commerce, les missions, les œuvres philanthropiques et éducatrices de l'Etat" (Revue de l'Institut de Sociologie, 1932, p. 701).

$51 \mathrm{Au}$ niveau scientifique, la logique du challenge global supplante celle de la capitalisation disciplinaire. "Les problèmes théoriques posés" : les mots traduisent un nouveau registre, à la fois parce qu'il y a "problèmes" et mieux, "problèmes théoriques". La variété et la nature des exposants lors de ces semaines montrent que la saisie de ces problèmes est d'emblée posée en objet de science, c'est-à-dire que les savants y gagnent comme savants une certaine légitimité à coté des "grands coloniaux". Les sciences ne se trouvent plus devant une série d'espaces nouveaux, mais face à un défi global. 
52 Tous les problèmes apparaissent liés et mieux, relèvent d'une seule problématique coloniale. C'est nouveau. Jusque là, il n'existait que des problèmes coloniaux à peu près disjoints, redevables de solutions techniques. Par ailleurs, rien dans les traditions scientifiques métropolitaines ne permettait d'annoncer le triple credo qui prend corps en matière de science coloniale : la colonisation doit passer sous contrôle scientifique, les sciences s'y font en conséquence sciences appliquées, l'objet colonial, plus que tout autre, suppose l'interdisciplinarité.

L'absence d'antécédent solide dans ces trois orientations et l'absolue démunition théorique et méthodologique des sciences sociales ne semble pas pour autant compromettant. La science prépare déjà la relève qu'elle assurera de la "Civilisation". La question coloniale n'atteint pas pour autant l'opinion publique.

Des thèmes qui se développeront dans l'après-guerre sont déjà bien présents. Entre autres, le grand programme de transmutation "de quelques millions de Noirs en paysans indépendants" qui motivera l'introduction des paysannats et plus tard, des centres ruraux expérimentaux aux mains des universités métropolitaines. (Leplae, professeur à l'Université de Louvain et Directeur Général au Ministère des Colonies. Revue de l'Institut de Sociologie, 1932, p. 769).

Dans le processus de problématisation savante sont aussi parfois contestés les dogmes les mieux établis de l'idéologie coloniale.

56 Un texte de Van Der Kerken, membre des sociétés royales savantes et professeur à l'Université de Gand ainsi qu'à l'Université coloniale, rend justice à de nombreux dénis concernant la culture ou l'infériorité des Africains, traite avec érudition de l'historicité des peuples du Congo. Relativisant déjà le fait ethnique, il évoque justement le fait linguistique bantou, décrit des "sociétés égalitaires démocratiques et collectivistes sans être communistes", traite des" sociétés aristocratiques". Il décrit positivement l'organisation sociale "bien adaptée à son milieu et relativement efficace," le contact actuel qui produit "une lente mutation des cœurs et des esprits", parie sur la capacité évolutive des sociétés africaines; "les indigènes restant profondément attachés à leurs institutions nationales, à leurs langues et traditions" et décrit en termes très actuels les problèmes de "communication" entre ces deux projets, problèmes très coûteux en sang et en argent. (Van Der Kerken, Revue de l'Institut de Sociologie, 1932).

Ce texte est remarquable. Avant même que le terme ne soit diffusé en Europe, il articule l'un des paradigmes scientifiques de la problématisation de la question indigène; l'acculturation.

En revanche la "bantouité" très fortement relativisée par V an Der Kerken se verra confirmée au plus profond du savoir colonial comme fait d'évidence débordant très largement le domaine linguistique. Comme en Union Sud-Africaine, il deviendra la formule à connotations pseudo-savantes mais en réalité essentiellement bureaucratiques, supplantant l''indigène". L'évidence culturelle et culturaliste "bantoue" régnera presque en maître absolu sur la problématisation coloniale jusqu'à son terme et ce, malgré d'épisodiques contestations ci et là. Pourtant, à l'opposé, nombre d'études ethnologiques entretenaient une vision atomisée des sociétés locales.

La période de guerre 1940-45 voit s'élever des voix d'administrateurs et de missionnaires qui dénoncent la violence de la mobilisation et du contrôle du travail aussi bien industriel que rural. Des études révèlent justement de véritables hémorragies démographiques dans certaines régions au moment même où l'on mesure 
immédiatement l'effet destructeur du refoulement vers la brousse des travailleurs surnuméraires. Par ailleurs les émeutes de Luluabourg de 1944 rappellent que la répression d'une grève du personnel indigène de l'Union Minière avait fait des dizaines de tués dès le début de la guerre, abattus par la Force Publique sous les yeux des autorités du Katanga venues entendre leurs doléances. De l'avis de tous, la guerre a été catastrophique pour 'Tindigène". C'est P. Ryckmans, Gouverneur Général de la colonie qui, dès 1946, réclame pour lui une participation aux fruits de la victoire.

Quelques spécialistes émergent au début des années 1940. Ils sont porteurs d'un autre discours, généralement réformiste et humaniste. A l'origine, ils ne s'inscrivent plus strictement dans l'espace traditionnel des sciences coloniales. Ils joueront un rôle-clé dans l'accélération de la problématisation de la question indigène qu'inaugure l'après-guerre.

61 Une figure telle que G. Malengreau (juriste, ethnologue de terrain et véritable fondateur de la première université au Congo) est caractéristique de l'alliage de savoir et d'humanisme réformiste qui participe de la saisie de la colonie par les sciences humaines.

62 Sa thèse conteste la notion de "terres vacantes" annonce ce que je désigne comme la problématisation tardive de la politique indigène, contribuant à mettre celle-ci au cœur de toute question coloniale. Ce moment est celui de la mutation des sciences coloniales qui quittent la stricte sphère de la capitalisation disciplinaire et des salons coloniaux pour se faire inspiratrices du traitement social de la question indigène. Malengreau écrit en 1947 que la "colonisation est au carrefour", que "la véritable mission d'émancipation est ... à commencer" et que "l'éducation politique des noirs doit supplanter l'ancien paternalisme". Pour d'autres, il s'agit de "jeter les bases d'une politique d'évolution appropriée". La conscience" critique" du système colonial, jusqu'ici portée par quelques juristes et missionnaires passe aux mains de jeunes intellectuels catholiques réformateurs: Ballegeer et Rubbens, en particulier au Katanga. Ils n'appartiennent pas aux cercles coloniaux métropolitains consacrés. On retiendra que ces deux là sont à l'origine du Centre d'Études des Problèmes Sociaux Indigènes en 1946. Rubbens est aussi l'auteur de "Dettes de guerre", ouvrage qui met à jour la créance du Congo sur la Belgique.

Ce moment dont nous ébaucherons ici seulement une esquisse me paraît fondamental à deux niveaux extrêmement imbriqués dans la pratique.

- La montée des sciences sociales et la restructuration du champ au bénéfice des universités.

Restructuration dont les effets sont toujours clairs aujourd'hui tant en termes de disciplines, d'institutions que de biographies.

- La problématisation du "progrès des indigènes" qui plante solidement le décor du développement et simultanément les infrastructures socio-scientifiques et paradigmatiques universitaires qui institueront les "savoir-développer" et présideront à leur reproduction. Jusqu'en 1956 la problématique sociale reste seule en lice. Mais brutalement un Plan de trente ans publié par un professeur de l'Université coloniale fait exploser le débat politique qui rebondit avec le Manifeste de Conscience Africaine, fruit d'un petit groupe d'intellectuels congolais. La décolonisation précipitée puis les événements congolais que je ne rappellerai pas ici, en focalisant l'attention sur les péripéties politiques feront l'impasse sur la politique sociale indigène, le bien-être, le développement... bref sur ce qui retient les "social activists". La machine (scientifique) à faire le développement est cependant lancée, elle poursuivra son œuvre. 
C'est dans l'immédiat après-guerre que se mettent en place les infrastructures de gestion de la "politique indigène". Apparaissent alors entre autres : un Plan Décennal de Développement du Congo, un Fonds du Bien-Etre Indigène, un Institut de Recherches Scientifiques pour l'Afrique Centrale (IRSAC) et une foule d'initiatives sociales et éditoriales.

L'évolution la plus importante sera l'institution et la multiplication des paysannats par l'administration coloniale sous contrôle scientifique de l'INEAC. L'objectif est vaste, il ne s'agit plus seulement de faire des recherches en station, mais d'une intervention directe et autoritaire visant la mobilisation du travail agricole. En réorganisant les régimes fonciers, en contrôlant de manière quasi policière la production agricole des produits d'exportation et le vivrier commercialisable, en réorganisant les instruments de commercialisation, il s'agit de "rationaliser" ce que tous les observateurs d'alors qualifient $d$ "'agriculture de rapine" de "semi-nomadisme", etc. Bientôt seront mises en pratique sous contrôle direct des agences créées par des universités métropolitaines les expériences de fabrication du "paysan véritable" ! Les paysannats offrent l'occasion des premières études scientifiques empiriques menées par des sociologues, ethnologues, géographes et agronomes non coloniaux et souvent issus des universités métropolitaines.

66 La "brousse" devient l'objet de tous les discours, on reconnaît qu'elle a connu durant la guerre "une régression catastrophique". Les études démographiques, alimentaires, sanitaires se multiplient. On évalue le potentiel piscicole des lacs, on importe des races bovines, etc. Le "sous développement" est virtuellement là même si le terme n'est pas utilisé. Il aurait été saugrenu en effet de qualifier le Katanga d'économie sous-développée. Cependant, l'amélioration du sort des paysans, le "progrès socio-économique de la brousse" devient un credo général mais surtout caractéristique des réformateurs qui condamnent par ailleurs les manipulations du système d'administration indirecte.

Par ailleurs, et nous retrouvons là typiquement la problématique des "classes dangereuses", l'immense mouvement de prolétarisation-migration-urbanisation a fait éclater l'espoir d'une stabilisation paisible de la main-d'oeuvre. La monétarisation des salaires, le développement d'entreprises destinées à capter ceux -ci, l'immigration devenue spontanée d'une région à l'autre et la "décrépitude des campagnes" entre autres thèmes, introduisent au temps fort de la problématisation ${ }^{4}$. Elisabethville et Léopoldville concentrent toute l'attention, le développement urbain y a été vertigineux. Léopoldville (Kinshasa) est passé de 116.000 habitants en 1946 à 390.000 recensés en 1956. A cette époque, il faut y ajouter des milliers de chômeurs non enregistrés.

La question sociale retiendra l'attention jusqu'à la problématisation politique très tardive. En fait, elle retardera celle-ci. L'esprit et la lettre de toutes les réformes d'intégration politique des congolais qui d'ailleurs n'aboutiront pas, témoignent de la permanence d'une approche qui soumet toute réforme de ce type à une promotion économique préalable.

L'ampleur de la question du prolétariat noir est sans commune mesure avec d'autres situations coloniales. En 1957, la seule "main d'œuvre indigène" dite "M.O.I." du Congo est quatre fois plus nombreuse que le total des salariés dans l'A.O.F. y compris les salariés blancs. (Enquête menée par l'ONU, chiffres donnés par Bouvier. 1965 p. 40). Ce 
n'est pas un hasard si ce sont le Katanga (Shaba) et Léopoldville (Kinshasa) concentrant ces masses hétéroclites qui deviennent les points de chute de cette politique sociale.

Outres les réformes administratives quant au statut des "évolués" ou des "détribalisées " ou "MOI" en général, on voit apparaître dans quelques entreprises des conseils consultatifs de la main-d'œuvre indigène ainsi que des ébauches de droits sociaux. Sur ces questions de droits sociaux, l'Institut de Sociologie Solvay se pose rapidement en expert (Doucy, Feldheim, 1954).

Une réforme du statut des villes trouve en 1957 un début de concrétisation après d'infinies discussions. Car toute volonté de réforme se heurte à la lourdeur administrative et au dogme que Young qualifie de "progressivité contrôlée", dogme selon lequel toute réforme doit commencer au niveau le plus infime être programmée sur la longue durée et enfin menée avec une incomparable prudence. A cette constante de la culture politico-administrative coloniale s'ajoute une autre certitude : tout vient de l'autorité, rien, absolument rien n'est attendu de la société indigène.

Sur ces différents points, il serait illusoire de croire que les spécialistes et experts universitaires se soient écartés de la problématique dominante.

Peu versés en tout cas dans l'ethnologie et ses subtiles distinctions, ils entérineront le plus souvent la "bantouité" dans ses expressions les plus banales. Le plus souvent aussi la science viendra tempérer l'ardeur réformiste.

74 Au Katanga minier, poumon financier de la colonie, apparaît le CEPSI (Centre d'Etudes des Problèmes Sociaux Indigènes) à l'initiative des anciens étudiants de l'Université catholique de Louvain. Il publie d'emblée un bulletin de niveau universitaire qui, sous différents titres, sera publié jusqu'en 1979. Le CEPSI aura une audience considérable. S'il témoigne de la particularité katangaise, il se veut d'emblée être l'élément moteur de toutes les initiatives et propositions de réformes sociales dans toute la colonie et au Ruanda Urundi. Au début, sous l'influence de jeunes catholiques et d'éléments de direction du grand capital, la politique coloniale de la métropole est très vivement critiquée. La promotion des Noirs, le changement des rapports sociaux entre Blancs et Noirs, la promotion de la brousse, sont les thèmes de choc.

Par le biais du CEPSI, Le grand capital colonial tente de promouvoir une gestion socialisée de la force de travail, c'est-à-dire d'y faire intervenir la Belgique comme Etat, ou à tout le moins, l'administration coloniale. La formation accrue de la force de travail est impérative dans la perspective de la rationalisation des outils de production... la formation et l'éducation deviennent donc impératives... pour le progrès de l'indigène. En centrant le regard sur l'organisation de la Cité, le logement, l'école et l'enseignement, les infrastructures, l'éducation des mères, le petit maraîchage urbain, les transports, etc., une "politique sociale" est ébauchée qui donne le change aux critiques du colonialisme belge, fait écho aux dispositifs d'intégration sociale en métropole et aux impératifs du grand capital minier mais surtout, dissimule l'immense processus de déstructuration socio-économique qui avait cours en fait depuis l'origine de "l'CEuvre". Je dis "dissimule" car les références à celui-ci resteront exceptionnelles dans le flot de littérature savante qui accompagnera cette mise en problématique. Au Katanga où règne le très fameux "Comité Spécial" souverain de fait et disposant de l'immense majorité des terres utiles, on s'interrogera poétiquement sur l'attrait des lumières de la ville à propos de la marginalisation définitive de "milieux coutumiers", ces corps exsangues qui ne réagiront même plus à quelques paysannats de choc. 
76 Néanmoins à l'origine du CEPSI, on trouve aussi l'activisme de jeunes intellectuels chrétiens progressistes dont les critiques à l'égard des ambiguïtés et erreurs commises dans la conduite des réformes ne tariront pas. A. Rubbens par exemple, à propos des procédures d'immatriculation des évolués (c. Young). On ne pourra pas en dire autant de certains universitaires liégeois et bruxellois qui prendront le pouvoir intellectuel à la colonie dans les années chaudes et finiront par se "mettre au service" de mouvements politiques locaux les plus opposés, parfois les moins crédibles.

Une autre revue apparaît elle aussi au Katanga: "Problèmes d'Afrique Centrale" à l'initiative des anciens étudiants de l'université coloniale (Anvers). Dès les premiers numéros est annoncé un nouvel impératif : "qui n'a pas l'esprit social ne doit pas faire carrière coloniale"! Elle fait elle aussi grand cas de l'expertise savante et son orientation globale témoigne du consensus qui s'établit rapidement, non sur la nature des réformes mais sur les contours du diagnostic en termes de problèmes sociaux. On y retrouve les spécialistes consacrés de la chose coloniale principalement issus de la haute administration et magistrature coloniale.

En 1947, la revue "Congo", jusqu'ici revue coloniale générale d'obédience chrétienne devient "Zaïre". Elle est souvent considérée comme la revue officieuse de l'administration de Bruxelles et prend elle aussi une orientation nouvelle en se faisant plus ouverte notamment aux universitaires non coloniaux, aux études menées par ceux-ci et par les nombreux "socio-ethnologues" spontanés que révèle l'agitation autour du nouveau problème indigène.

79 L'Institut Royal Colonial Belge redouble d'activités et de publications relatives aux paysannats et aux "centres extra-coutumiers", "cités" et "camps de travail" qui, soigneusement séparés des villes blanches, concentrent les travailleurs mais aussi les "clercs" (employés noirs des échelons inférieurs de l'administration et du commerce) et accueillent le mouvement d'exode rural.

80 La plupart des centres extra-coutumiers font l'objet de monographies et on peut dire que celui d'Elisabethville devient un véritable champ d'expérimentation sociale (Grevisse, 1951, Benoît, 1959, Minon, 1960, Dethier, 1961).

81 Des fiefs universitaires s'y constituent à l'initiative et sous le contrôle de l'UMHK. Les instituts de sociologie de Liège et de Bruxelles y dirigeront d'abord des foyers sociaux et des centres éducatifs, chantiers de jeunesse, foyers féminins, etc. Un des premiers chercheurs se souvient "le personnage du chercheur en sciences humaines est inédit et incompris dans le Katanga de cette époque". Mais il est là !

L'Institut Royal Colonial International qui devient l'INCIDI (L'institut International des Sciences Politiques et Sociales Appliquées aux Civilisations Différentes) n'est pas en reste et sa mission de légitimation trouve avec le "social" de nouveaux terrains de réflexion. Les thèmes des sessions en témoignent: les classes moyennes dans les colonies, les classes paysannes, les classes ouvrières, l'éducation, la propriété indigène. Durant les années 1950, on trouve parmi les membres des personnalités telles que Balandier, Herkovits...

83 Il n'est pas abusif d'avancer que tout le champ savant et éditorial colonial se transforme et témoigne de la transition vers la guidance scientifique du progrès de la société indigène. Il est cependant évident que le credo cache mal la quasi absence de nouveaux référents théoriques. L'accent est donc sans cesse porté sur la méthode qui constitue le propre d'une science qui "saisit enfin ces questions". 
Complémentairement à cette fixation sur la méthode, c'est l'interdisciplinarité qui est érigée en garantie suprême. La question coloniale tardive est donc reconstruite comme un phénomène social total. Les sciences sociales se distinguent essentiellement par une constante insistance sur le primat de la mutation humaine en cours. Un primat d'ailleurs largement reconnu par la plupart des scientifiques. Le credo supplée à l'absence théorique et à la maigreur des connaissances empiriques.

Les universités pour leur part créent des programmes spécifiques de formation coloniale. Dès 1947, l'Institut Solvay lance une "Série Coloniale" rompant le monopole des sociétés savantes nationales et spécifiquement coloniales. L'Institut du Travail de l'ULB crée une section coloniale délivrant un titre dont on devine qu'il devait anticiper le marché colonial professionnel de l'inspection légale du travail au Congo. Nul ne doute alors que les réformes, extrêmement progressives évidemment, seront apparentées à celles que connut la métropole.

6 Les universités prennent donc pied sur le marché de la question sociale indigène de diverses manières. Le Congo devient d'autant plus objet d'investigations que les autorités se refusent à accueillir les missions des organismes internationaux de contrôle et entendent produire leur propre information.

À partir de 1955, les dispositifs d'intervention directe des universités s'étoffent considérablement. Si Louvain se concentre sur le développement de Lovanium (première université du Congo), c'est que le dispositif missionnaire qui reste très puissant sur le terrain est un débouché et un relais incomparable. Depuis 1956, l'Eglise a pris l'initiative de rompre publiquement le consensus de la trinité coloniale en se désolidarisant de l'administration. Cette prise de position décisive est aussi une réplique aux libéraux laïques qui tentent de briser le monopole religieux sur la production des élites congolaises.

L'ULB qui dispose d'un outil essentiellement médical (CEMUBAC) au nord-est de la colonie, étend ses activités au Katanga avec des foyers sociaux et un centre de formation sociale. Les mandats coloniaux se multiplient et des chercheurs apparaissent dans les villages et les cités. L'Université de Gand dispose d'un outil également avec la Fondation Ganda-Congo.

89 L'Université de Liège, en reste jusque là, entreprend de rattraper le temps perdu et, grâce à des accords avec le CEPSI, s'installe dans les cités extra-coutumières d'Elisabethville (Lubumbashi). L'UMHK investit ainsi indirectement dans la recherche sociale une infime partie des bénéfices plantureux réalisés durant la guerre de Corée. $\mathrm{L}$ 1nstitut de Sociologie se dote d'une division Afrique dont le credo sera le service social qui se systématisera en "développement communautaire". Les recherches sont menées tambour battant et le service social s'étoffe sur base du thème de la participation et de la valeur d'exemple. Les deux foyers d'action retenus sont la famille (conjugale s'entend) et le travail à travers la formation professionnelle. Le petit maraîchage urbain à vocation éducative et stabilisatrice fait partie de l'arsenal (Dethier, 1961). Les services sociaux sont mis en place alors que des recherches socio-démographiques, alimentaires. psychosociologiques commencent. Dans un deuxième temps, la recherche-action sera privilégiée. à l'instar des méthodes qualitatives d'investigation.

Une fondation ad hoc (FULREAC, Fondation de l'Université de Liège pour les Recherches Scientifiques au Congo et au Ruanda Urundi) est à l'origine de ce qu'on peut qualifier de premier projet de développement rural intégré. Le centre rural expérimental FULREAC 
I doit faire la démonstration suivante : la "brousse" est économiquement viable pourvu que l'exploitation agricole soit rationalisée. Mieux, la "brousse" doit pouvoir être aussi attractive socialement que les villes. On espère ainsi" animer l'hinterland", amorcer une autonomie régionale vivrière, mais aussi renverser le mouvement d'exode rural et surtout réinstaller des néo-citadins encombrants. Dans cette perspective, des cités satellites ont été envisagées comme lieux de transit dès 1955 par le CEPSI. A Léopoldville, des chantiers de travail agricole sont destinés à occuper un nombre croissant de chômeurs. Dès 1956, le chômage devient en effet le problème numéro un.

Le centre rural expérimental est cependant conçu comme un village disposant d'un encadrement scolaire directement inspiré du projet productif, de services éducatifs divers, de structures coopératives et communautaires, de dispositifs de crédits, de prêts de matériels, etc.

Derrière ces deux interventions liégeoises, on trouve in fine l'UMHK (Union Minière) et ses agences sociales. Ici plus qu'ailleurs, et peut-être en raison du caractère tardif de cette intervention, se révèle presque caricaturalement l'idéologie scientifique se proposant la relève de la civilisation. Ici on fabrique "l'homme nouveau" sous contrôle scientifique. Le credo des universitaires qui s'initient à l'action concrète est alors l'interdisciplinarité, le désintéressement et la neutralité académique.

93 L'UMHK, dont les filiales recouvrent toute l'économie du Katanga, cherche pour sa part à "faire du social" sans trop apparaître comme telle sur la scène publique coloniale.

94 Sans rompre avec les principaux fondements de l'idéologie coloniale on proclame la relève scientifique. On se fonde sur ce constat: "jamais la colonie n'a bénéficié d'une équipe multi-disciplinaire agissant en toute indépendance sur le terrain"(Dubuisson, Recteur de l'Université de Liège).

95 Ce sont des missions interdisciplinaires liégeoises qui aboutissent à la mise sur pied du centre rural expérimental. On recrute des "futurs paysans" qui seront globalement encadrés par des agronomes, des sociologues, pédagogues et des psychologues. Un enseignement technique agricole est mis sur pied pour les enfants, de même qu'un encadrement socio-éducatif des mères.

Le centre expérimental, qui sera très vite rentable, se définit comme une coopérative sous contrôle scientifique. Mais on abandonnera tout aussi vite ce principe pour le salariat. Cette activité se poursuivra lors de la sécession katangaise et les nostalgiques de l'ordre "communautaire-coutumier", ne renonceront pas au principe coopératif alors défaillant On s'intéressera de près aux Kibboutz pour y trouver quelques solutions. Mais il s'avérera difficile de lutter contre un "turn over" lié à l'insécurité du moment Aux yeux des promoteurs, le salariat est contraire au principe de la "coutume" et surtout, il introduit des formes d'organisation collective jugées suspectes. On répète qu'il ne s'agit en aucun cas de fabriquer des prolétaires. Cette critique avait d'ailleurs déjà été faite aux paysannats. La formule de ceux-ci est d'ailleurs explicitement rejetée par les promoteurs de FULREAC qui prônent la participation plutôt que la mobilisation, l'éducation plutôt que la menace, l'enseignement professionnel adapté plutôt que l'enseignement généraliste missionnaire, etc.

97 La pédagogie scientifique mise en œuvre se réclame de Freinet! Il faut "désoccidentaliser d'urgence" l'enseignement qui produit ces êtres superficiels que sont les évolués ! 
Les expériences liégeoises au Katanga, caricaturales peut-être parce que tard venues, constituent à mes yeux le prototype du projet savant. Il vire d'emblée à l'utopie technocratique, autoritaire voire totalitaire. L'indigène n'y est défini que comme une somme de lacunes, de défauts. Il est mobilisé, à peine sollicité dans ses caractéristiques propres fussent-elles imaginaires.

Incontestablement, les sciences sociales qui font plus que jamais profession de scientificité projettent sur ce nouvel espace imaginaire (le développement) la structure idéale de la démarche hypothético-inductive qu'elles vénèrent. A de nombreuses reprises transparaît une analogie curieuse entre la méthode scientifique et la méthode proclamée de "guidance du changement social". Il s'agit probablement d'une confusion qui dépasse la simple analogie.

Par ailleurs, apparaît une critique relative de la colonisation. J.-P. Harroy, pionnier de l'environnementalisme et de l'écologie coloniale, Secrétaire Général de l'IRSAC, futur Vice-Gouverneur puis Résident Général au Ruanda Urundi, dénonce la dégradation des sols due à des pratiques coloniales longtemps fondées sur l'idée répandue de l'inépuisable fécondité des sols africains. Quelques sociologues et psychologues dénoncent la ségrégation de fait alors que le discours officiel met un point $\mathrm{d}$ 'honneur à bannir le "color bar". Le thème de l'amélioration des "relations humaines" est en effet au centre des discours réformistes. Mais le cadre de référence de ces critiques reste lié à l'idéologie coloniale et ses a priori: bantouisme, retard des sociétés africaines, sous-productivité du travail et bien sûr, mission émancipatrice de la science. Il faudra attendre les années 1960-65 pour que, dans la foulée de l'ouvrage "ravageur" de Merlier (1962), une critique radicale s'appuyant sur des concepts marxistes rompe le cercle vicieux en décentrant toute la problématique de départ (Demunter, 1975).

1 Revenons en milieu urbain durant les années 1950. Dans les cités de travailleurs, un important travail de recherche est mis en place à partir des foyers socio-éducatifs. Les monographies quantitatives des populations succèdent aux relevés urbanistiques. Très vite apparaît l'exigence du qualitatif et des travaux originaux verront le jour qui utilisent l'investigation informelle, l'observation directe et participante (Dethier, 1960 ; Richelle, 1961). Le débouché naturel en sera le développement communautaire dont la philosophie est donnée par un petit texte publié en 1960 (Service social et développement communautaire, 1960).

Dans ce cadre comme dans le cadre des centres ruraux, l'acculturation devient le paradigme dominant des recherches qui sont de type psycho-sociologique. Elles traduisent bien sûr la réception des travaux d'anthropologie culturelle de langue anglaise mais sont aussi l'aboutissement de l'évolution de la "science pratique" des coloniaux. Aux yeux des observateurs de l'époque, l'acculturation, bien qu'amorcée, devait passer sous contrôle scientifique pour devenir un facteur de développement et d'ordre. Dans un premier temps, l'approche de l'acculturation reste sans rapport avec les principes du relativisme culturel.

3 La thèse de $\mathrm{M}$. Richelle consacrée à l'acculturation en milieu urbain fait suite à des recherches menées en 1958 dans la foulée de l'établissement du centre expérimental FULREAC I. En recourant massivement mais de manière critique à l'anthropologie culturelle et à la représentation culturaliste, elle fait apparaître la tardive capacité de rupture du discours savant par rapport à l'idéologie et aux sciences coloniales. Le relativisme fonctionnaliste, faut-il le rappeler, est alors une révolution épistémologique. Il était temps, nous sommes en 1960 ! 
Certains si embarrassés qu'ils étaient de cette révolution qui invalidait peu ou prou leurs savoirs, prennent très vite la mesure de l'événement alors que se termine l'ère coloniale et que s'ouvre l'ère de la coopération. C'est l'anthropologie culturelle qui triomphe. Voici, résumé en une formule, l'essentiel de cette révolution: "En quoi et pourquoi ces gens diffèrent-ils de nous? On a invoqué tour à tour le milieu géographique, l'environnement tropical, le régime nutritionnel, et surtout la race. Tout cela est secondaire. C'est l'anthropologie culturelle, c'est la notion de culture qui fournit la réponse" (Paulus, psychologue et professeur à l'Université de Liège, préface à Richelle, 1960). Pour le langage commun, "c'est sur cet homme noir que nous connaissons si mal que reposera désormais le futur de notre œuvre".

À travers ces derniers mouvements des savoirs proprement coloniaux, les sciences sociales n'ont cessé de se donner une mission pratique. Cette démarche n'était pas courante en métropole. Au Congo, les sociologues et psychologues se font jardiniers! Les plus téméraires se risquent dans les buvettes des cités! Les habitations et leurs contenus font l'objet de relevés exhaustifs. Des échantillons sont prélevés, etc. Mais les activistes du social donnent aussi des cours, aménagent des locaux, planifient des villages, animent des groupes d'orphelins, etc. Jamais peut-être des chercheurs n'auront été impliqués de la sorte et aussi directement dans ce qu'ils croient être le travail d'accouchement d'une société nouvelle.

De cette saisie scientifique $d u$ fait colonial tardif qui met en place le développementalisme dans un contexte d'urgence, l'économie comme discipline est singulièrement absente.

107 En fait et aux yeux de tous l'économie (comme réalité), le "développement économique" est un donné. Il est donné par le fait de l'industrialisation massive et ses modalités si spectaculaires au Congo. Son étude reste du domaine du grand capital, des grandes entreprises, et de l'Administration dans une moindre mesure. Au Ruanda Urundi on ne quitte pas les domaines de l'aménagement de l'économie agro-pastorale et du bien-être indigène rationalisé.

La première étude macro-économique considérant le Congo comme une unité significative date de 1955 sous la plume de F. Bezy. Il s'agit d'une approche structurale de l'économie de la colonie.

Des écrits à caractère économique, la notion de "sous-développement" est littéralement absente. On parle plutôt de déséquilibres. De l'avis de l'auteur, "il n'y a rien à l'époque (1955) sur la notion de sous-développement. Un texte de Lewis peut-être, mais cela arrivait en retard au Congo"(entretien avec F. Bezy). Dès 1949, un plan de développement économique du Congo avait pourtant été lancé.

110 Les économistes sont très discrets dans les missions savantes et prennent peu part aux forums du réformisme colonial. Ils ont peu à dire à propos de l'économie coloniale. Dans l'esprit des réformistes, l'économie en elle-même ne pose pas problème. Dans les programmes "coloniaux" des universités, l'économie coloniale est à peine enseignée.

111 Domaine réservé ? Il est vrai que malgré les crises périodiques, la colonie vit une espèce d'euphorie de croissance depuis quarante ans. L'économie n'est donc pas centrale dans le processus de problématisation qui annonce le développement Pourtant en 1958-59, il est admis que le Congo relève de l'économie sous-développée ! Coup de force ? Coup de génie? La performance conceptuelle et théorique de l'économie universitaire est d'autant plus remarquable qu'elle était en retard et que son matériau lui restait 
exogène, qu'elle ne disposait pas d'entrées empiriques. Dès 1958-59, elle proposera pourtant une grille d'analyse inédite et structurale du "sous-développement". On invoque alors l'économie moderne et l'auteur cité ci-dessus peut faire référence à celle-ci pour tracer le balisage critique du second plan de développement alors envisagé pour le Congo (Bezy, 1959). L'économie est alors capable de produire un discours fondant des prescriptions sur un apparent background théorique. Cette performance sera d'autant plus importante que le professeur Bezy sera à l'origine de l'IRES-Lovanium qui deviendra le principal centre de recherches en sciences sociales au Congo.

112 C'est l'IRSAC (qui ne comprend pas de section économique) créé en 1947 qui fournit le lieu de naissance de cette ethnologie universitaire. L'IRSAC participe évidemment de la relève scientifique de la "Civilisation". Il est conçu dans la perspective d'un progrès accéléré de la colonie et des territoires sous tutelle, progrès dont il doit être un moteur. Il s'agit donc d'un micro-site capital pour la construction et l'institutionnalisation des savoir-développer. A la fondation de cette institution qui entend couvrir toutes les matières scientifiques, il n'y pas d'ethnologie africaniste professionnelle belge. Jusqu'ici, et malgré le précédent du Mouvement Sociologique, elle reste le fait de missionnaires et de quelques administrateurs. On la crée donc. L. De Heusch est en formation chez Griaule; Biebuyck et Maquet sont prestement envoyés auprès de D. Forde à Londres. La section d'ethnologie basée à Astrida (Ruanda Urundi) sera, outre les précédents, une pépinière d'ethnologues de renom: Vansina, Coupez, D'Hertefelt, Doutreloux, etc.

113 Des travaux importants d'anthropologie physique seront menés par Hiernaux. Harroy est Secrétaire Général de l'institution, et ses travaux sur le Ruanda-Urundi en feront un pionnier de l"écologie coloniale" et du conservationnisme. Les sociologues sont absents de l'IRSAC.

114 De remarquables travaux linguistiques notamment entrepris par des missionnaires-ethnologues spontanés seront également subventionnés par l'IRSAC.

115 La première ethnologie professionnelle universitaire belge qui s'exportera d'ailleurs remarquablement après les indépendances, participe donc de cette mise en problématique de la question indigène. Elle restera cependant très éloignée des soucis et des terrains des activistes du social. Tard venue, elle s'oriente d'emblée sur les normes et institutions internationales de la corporation.

Nous ne traiterons pas ici de la problématisation politique de la fin du fait colonial sinon pour signaler qu'elle mobilisera de nombreux chercheurs à la suite de P. Caprasse et $\mathrm{P}$. Bouvier et qu'elle trouvera elle aussi son paradigme : la modernisation.

117 La précipitation de la décolonisation et ses avatars ultérieurs donne ainsi une prime aux approches du CRISP (Centre de Recherche et d'Information Socio-Politique à Bruxelles). La série chronologique restituant dans le détailles événements congolais sera remarquable de ce point de vue, de même que des publications d'analyse qui prennent rapidement la mesure de l'événement (J.-J. Libois, C. Young).

À Lovanium, au Congo donc, les dernières années de la colonisation voient donc naître à l'initiative de F. Bezy l'Institut de Recherche Economique et Sociale (IRES) qui comportera trois sections : anthropologie, économie et sociologie. Au lendemain de l'indépendance, l'IRES sera le principal foyer de recherche sur le Congo et le second pôle de décolonisation savante tout en étant de fait le principal fournisseur des 
gouvernements congolais en matière d'information économique et sociale. L'IRES aura bientôt son alter ego louvaniste avec l'Institut des Pays en Développement, dirigé par Bezy et Malengreau, et qui s'enrichira considérablement de contacts précoces avec l'Amérique latine.

Doucy, Bouvier et Harroy parmi d'autres, réimporteront le "développement" à l'ULB, à travers notamment un Centre d'Etudes des Problèmes de l'Industrialisation de l'Afrique Noire et la création d'un cycle universitaire complet en sciences sociales spécifiquement axé sur les PVD.

Quant à L'Université de Liège, elle se remettra mal de l'engagement de certains de ses "héros coloniaux" de la onzième heure aux côtés de Tshombe et de la sécession du Katanga. Néanmoins, et quelque soit le poids de quelques engagements passés de certains de ses membres, les initiatives se multiplient à la suite de l'indépendance pour élargir le domaine des enseignements relatifs aux dorénavant PVD, pour reprendre pied sur les marchés de l'expertise et de la coopération. Sous la sécession katangaise, les "bruxellois" ont été écartés de l'Université d'Elisabethville qui passa aux mains des "liégeois". Une tradition de coopération universitaire se nourrit donc parallèlement au tandem Lovanium-Louvain.

121 À la veille de la décolonisation, plus de 500 chercheurs scientifiques étaient en activité au Congo et Ruanda Urundi (Van Hove, 1968). Quelques-uns deviendront coopérants ou intégreront le "Ministère de la Coopération". D'autres, de retour, grossiront les rangs des experts en "choses du développement" et tiendront le haut du pavé des nouvelles sciences du développement jusqu'à l'émergence d'un pôle récent issu des activités des ONG. Les programmes universitaires en sciences sociales feront large place aux arts et sciences du développement. Les universités ont définitivement "enlevé" le "développement" qui échappe aux cercles savants coloniaux fortement atteints par les événements ultimes. Les spécialistes ès-Congo élargissent dorénavant leurs vues à l'échelle du Tiers-Monde (voir Doucy. 1970).

Les quinze années qui suivent la seconde guerre mondiale auront donc été celles de l'invention d'un objet savant nouveau, de la définition d'approches théoriques et méthodologiques, de la définition d'un terrain vite élargi au Tiers-Monde. Invention tout autant d'objets d'enseignement, de recherche et surtout, de pratiques et de registres de compétences qui, une fois réimportés en métropole, vont trouver immédiatement place dans les marchés savants que suscitent la mondialisation du "développement", les nouvelles pratiques de coopération et la concrétisation du Tiers-Monde.

Mais plus encore, la "Civilisation" se faisant discrète, "l'Cuuvre" étant brutalement démonétisée, la pratique scientifique est dorénavant en mesure d'ajouter un appareillage institutionnel, un complexe de spécialisations et de pratiques à ce qui n'est resté longtemps qu'un credo. Durant ces quinze années, des organismes internationaux comme l'UNESCO ont contribué à internationaliser la réflexion. Mais toute la logique des sciences coloniales allait déjà dans le même sens et les chercheurs belges s'y sont inscrits d'emblée. Les forums internationaux ainsi constitués faisant large place aux sciences de l'homme, ont produit, ou à tout le moins diffusé, de nouvelles thématiques définissant de nouveaux profils de compétence: développement communautaire, développement coopératif... De véritables "entreprises d'expertise et de management en développement" voient le jour dans ce nouvel espace. Celle du R.P Lebret est 
caractéristique à cet égard et est peut-être la première multinationale dans ce domaine qui supplante des organismes comme l'INCIDI.

La poussée des sciences sociales va bouleverser le vieil ordre des sciences coloniales et proposer le double programme de saisie théorique du changement social et d'ingénierie du Progrès. Paradoxalement, les "social scientists" dénoncent aujourd'hui la portion congrue que leur réservent les marchés du développement !

On peut se demander si ce credo des sciences sociales et les théories de la modernisation qui vont suivre n'ont pas élargi de manière inouïe le mythe "manheimien" d'une classe d'intellectuels sans attache" (freichwebende intelligenz), étendue cette fois à la planète, réconciliée et liquidant le colonialisme, transcendant la diversité culturelle et affrontant un défi qu'elle énonce elle-même comme "colossal et historique".

Le développement a fourni une extraordinaire caisse de résonance à une ambition, un credo "scientiste" tout autant qu'à des connaissances établies. C'est évident au regard des investissements du terrain colonial tardif par les sciences sociales belges.

Par ailleurs, nul autre espace idéologique n'a été aussi propice à une translation des "savoirs savants" dans les idéologies et pratiques quotidiennes. La structure polémique notamment manifestée par les courants dépendantistes, loin de disqualifier l'ensemble de ce processus, a joué pour lui un rôle catalyseur. Absent pour des raisons identifiées, le développement n'en devenait que plus présent Le "développement" relevait désormais de la Raison, fut-ce et peut être surtout, sur le mode polémique des prédations Nord-Sud.

128 Le retour sur soi qui caractérise nombre d'entreprises scientifiques actuelles ou l'auto-archéologie transparaissant de la pléthore actuelle de manuels en langue anglaise qui tendent à reconstruire ces disciplines du "développement" selon un modèle quasi canonique où se succèdent les temps de la "modernisation enthousiaste", de la "critique structuro-dépendantiste", de "l'éclatement des problématiques" et enfin de la "spécialisation et de la sectorialisation", suggèrent insidieusement une logique d'accumulation dialectique qui rappelle finalement l'idéale progression de la "Science" (Barnett, 1988). Par ailleurs, les inflexions et oppositions théoriques y sont trop souvent simplement reliées à quelques grands événements internationaux: Truman et sa déclaration d'investiture de 1947, Bandung, Boumédienne à l'ONU, etc. Ils négligent les foyers locaux principalement coloniaux ou métropolitains européens et leurs processus de mise en réseaux à la veille des indépendances. Voir à titre d'exemple Forde, 1956, ouvrage collectif où les questions congolaises sont abondamment traitées par des universitaires belges et étrangers. Les infrastructures socio-scientifiques, les délimitations d'objets, les capitaux symboliques, les réseaux de chercheurs et de décideurs sont bien en place avant les indépendances pour conquérir les marchés savants du développement.

Les pionniers des sciences du "développement" au Congo ne disposent pourtant d'aucun corpus théorique sur lequel appuyer leurs propres constructions, ils inventent une pratique et un discours, amalgame curieux d'idéologie coloniale, d'ethnographie pratique, de réminiscences de l'histoire européenne récente, fruit de circonstances locales autant que d'urgences internationales. On leur prête volontiers car s'installe une nouvelle idée qui veut que "l'Homme soit la clé de tout mieux-être possible". 
Entre la logique kuhnienne du paradigme et le "everything works" de Feyerabend, un espace se dessine donc pour une sociologie circonstanciée de la production savante et de la reproduction du "développement". Celui-ci étant devenu outre une doxa, une "évidence contraignante" pour les nouveaux acteurs, cette sociologie de la connaissance savante devrait se doubler d'une sociologie de la connaissance pratique, quotidienne, telle qu'elle retient principalement l'attention de l'interactionnisme symbolique. On imagine bien l'étude des mises en scènes quotidiennes du "développement".

131 Mais nous n'aborderons pas ici cet aspect de l'archéologie du développement. Rendons à César...

132 Le "développement" est spécifiquement et originellement un produit savant, c'est-à-dire qu'il renvoie à des logiques précises situées dans des espaces sociaux bien particuliers qui se sont constitués dans des contextes historiques précis. A trop dénoncer la boulimie et la vulgarité des consommateurs, n'oublierait-on pas les producteurs?

\section{BIBLIOGRAPHIE}

L'Année Sociologique, Vol. 42, Sociologie du développement, bilan et perspectives. (En particulier les contributions de R. Boudon, A. Guichaoua et A. Touraine).

Arndt H.W., Economic Development. History of an Idea. Chicago University Press, 1988.

Barnett T., Sociology and Development.

London-Melbourn-Sydney-Auckland-Johannesburg-Hutchinson, 1988, 239 p.

Boirai P., Lantéri J.-F., Olivier de Sardan J-.P., Paysans, chercheurs et experts en Afrique noire. Sciences sociales et développement rural. Editions Karthala/Ciface, 1985.

Boudon R., La place du désordre. Paris, P.U.F., 1984.

Cardoso F., Les idées à leur place. Le concept de développement en Amérique latine. Paris, Editions Métaillé, 1984.

Dube S.C., Modernization and Development : The search for Alternative Paradigms. Tokyo, Zed Books LTD/Université des Nations-Unies, 1988.

Forde D. sous la dir., Aspects sociaux de l'industrialisation et de l'urbanisation au sud du Sahara. Etude réalisée sous les auspices de l'UNESCO par l'Institut International Africain. Paris, UNESCO, 1956.

Girardet R., L'idée coloniale en France. 1871-1962. Éditions de la Table Ronde, 1972.

Goussault Y., Tiers-Monde, développement : de la socio-économie à la sociologie, Revue Tiers Monde (Paris), t. XXVIII, n 112, oct.-déc. 1987, pp. 759-776.

Goussault Y., Guichaoua A., La sociologie du développement, in J-.P. Durant et R. Weil, Sociologie contemporaine. Paris, Editions Vigot, 1989, pp. 759-776. 
Hugon P., La pensée française en économie du développement, Revue d'Économie Politique, 101 (2), mars-avril 1990, pp. 171-229.

Harrison D., The sociology of modernisation and development. London New York, Routledge, 1988196 p.

International Sociology, Vol. 7, $\mathrm{N}^{\circ} 2$, The future of Development.

Leclerc G., L'observation de l'homme. Une histoire des enquêtes sociales. Éditions du Seuil, Paris, 1979.

Marseille J., Empire colonial et capitalisme français. Histoire d'un divorce. Éditions du Seuil, Paris, 1984.

Meier G.M., Dudley S. (Ed), Les pionniers du développement. Paris, Éditions Economica pour la Banque mondiale, 1988.

Piault M.-H. (sous la dir.), La colonisation : rupture ou parenthèse ?, Paris, Éditions L'Harmattan, 1987,326 p.

Poncelet M., Culture et développement : des objets à reconstruire. Mémoire de troisième cycle, Louvain, 1990.

Sachs W., L'archéologie du concept de développement, Inter Culture, Vol. XXIII, 1990, nº 4.

Sachs W. \& al., The development Dictionary. London, Zed Books, 1991.

Revue Tiers-Monde, parmi les numéros récents : 1982, Tome XXIII, $\mathrm{n}^{\circ} 90$ : Sociologie du développement ; 1984, Tome XXV, nº 97, Culture et développement ; 1984, Tome XXV, $\mathrm{n}^{\circ}$ 100, Le développement en question; 1987 . Tome XXVIII, $n^{\circ} 112$, Les débats actuels autour $d u$ développement.

Webster A., Introduction to the sociology of development. Londres, Mac Millan, 1990.

Worsley P., The three Worlds. Culture and World Development. Chicago, The University of Chicago Press. .

Bibliographie sommaire des arts et sciences du développement au Congo et en Belgique

Benoît J., La population africaine à Elisabethville en 1957. FULREAC/CEPSI, Elisabethville, 1959.

Bezy F., Problèmes structurels de l'économie congolaise. Éditions Nauwelaert. Louvain 1957.

Bezy F., Principes pour l'orientation économique au Congo. Problèmes sociaux congolais, 1959, $\mathrm{n}^{\circ}$ 47, pp. 97-121.

Bouvier R.P., L'accession du Congo belge à l'indépendance. Essai d'analyse sociologique, Bruxelles, Editions de l'Institut de Sociologie de l'Université Libre de Bruxelles, 1965.

Caprasse P., Leaders africains en milieu urbain. Mémoire du CEPSI, Elisabethville, 1959.

Clemens R., Fondements sociologiques de l'action sociale en Afrique belge. Problèmes sociaux congolais, $\mathrm{n}^{\circ} 47,1959$.

Clemens R., La mentalité d'entrepreneur aux premières phases du développement. Actes du 5 e congrès mondial de sociologie. Washington, International Sociological Association, 4 t., 1. 2, 1962, 208 p., pp. 33-50.

La Conférence Géographique de 1876. Académie Royale des Sciences d'Outre Mer, Bruxelles, 1976.

De Bie P., Naissance et premiers développements de la sociologie en Belgique. CIACO, Louvain la Neuve, 1988. 
Demunter P., Masses rurales et luttes politiques au Zaïre. Le processus de politisation des masses rurales au bas-Zaïre. Editions Anthropos, Paris, 1975.

Doucy A., Introduction à l'économie sociale du tiers-monde. Bruxelles, Institut de sociologie, 1970.

Gourou R., La densité de population rurale au Congo belge. ARSC, classe de sciences naturelles et médicales, Mémoire in $8^{\circ}$, t. I, fasc. 2, 1955.

Dethier R., Une famille de citadins du Katanga, Collection des travaux de l'Institut de sociologie. Université de Liège, Liège, 1961, $\mathrm{n}^{\circ} \mathrm{XI}$.

Dethier R., Les citadins katangais et leurs jardins. Aspects psycho-sociaux de la vie en milieu africain urbain, Collection des travaux de l'Institut de sociologie de l'Université de Liège, Liège, $1961 \mathrm{n}^{\circ}$ XIII.

Développement communautaire et service social en Afrique. Premiers pas de l'action sociale à Katuba, Elisabethville, Institut de Sociologie de l'Université de Liège, Liège 1960.

Doucy A., Feldheim P., Problèmes du travail au Katanga, Revue de l'Institut de Sociologie (ULB) $\mathrm{n}^{\circ} 3$, 1954, pp. 393-416.

Vers la promotion de l'économie indigène. Études coloniales, fasc. III Compte rendu du colloque sur l'économie indigène. ULB, Solvay 1956.

Grevisse F., Le centre extra-coutumier d'Eville. Publication de l'IRCB, CEPSI/Institut Royal Colonial Belge, 1951, $\mathrm{n}^{\circ} 15$.

IRSAC, Rapports annuels d'activité, 1947-1960.

Malengreau G., Le Congo à la croisée des chemins, la Revue nouvelle, janvier 1947.

M'Bokolo E., "Le Congo. Colonie modèle", in Conflit belgo-zaïrois, fondements historiques, politiques, économiques et culturels. Présence africaine, Paris Dakar, 1990.

M'Bokolo E., "Le séparatisme katangais", in Au cœur de l'ethnie. Ethnies, tribalisme et Etats en Afrique, sous la direction de J.-L. Amselle et E. M'Bokolo. Editions la Découverte, Paris, 1985.

Merlier M., Le Congo de la colonisation belge à l'indépendance, Éditions Maspéro, Paris, 1962.

Minon M., Katuba, Étude quantitative d'une communauté urbaine africaine. Collection des travaux de l'Institut de Sociologie ${ }^{\circ}$ VIII, Université de Liège, Liège, 1960.

Le Congo, compte rendu de la semaine sociale. La Revue de l'Institut de Sociologie, $n^{\circ} 4,1932$, Bruxelles, Institut de Sociologie Solvay.

Richelle M., Aspects psychologiques de l'acculturation. Recherche sur les motivations de la stabilisation urbaine au Katanga. Editions Fulreac, 1961.

Richelle M., Le centre rural expérimental Fulreac I. Réflexions sur les problèmes humains au terme d'une année de fonctionnement, Editions Fulreac, Liège, 1960.

Stengers J., Combien le Congo a-t-il coûté à la Belgique ?, ARSC, Classe des sciences morales et politiques, Mémoire in 80, nouvelle série t. XI, fasc. l, Bruxelles, 1957.

Vanderlinden J., La crise congolaise, Éditions Complexe, Bruxelles, 1985.

Van Hove J., Histoire du Ministère des Colonies. Académie royale des sciences d'outre mer, Bruxelles, 1968. 


\section{NOTES}

1.J'ai essayé d'appliquer ailleurs la formalisation webero-bourdieusiennne du marché des biens de salut au champ du développement. Cf. Poncelet, 1991.

2.Ce constat permet d'aborder autrement la question de l'expertise fournie par les sciences sociales aux développeurs. Car il est tout à fait illusoire de poser cette question en faisant comme si on avait d'une part une demande "profane" et de l'autre un corps de connaissances et de méthodes indépendantes de la première, Une des caractéristique du champ des savoir-développer concerne en effet ses liaisons inextricables avec l'idéologie et les pratiques du "développement" et par ailleurs son caractère excentré sur le plan scientifique et académique.

3.Cette tentative sera rééditée quelques quarante années plus tard par l'IRSAC (Institut de Recherches Scientifiques pour l'Afrique Centrale fondé en 1947) comme nous le verrons dans la suite.

4.D'immenses régions sont en fait en proie à une politique de pillage de la part des grands groupes d'intérêts industriels régnant de fait sur des centaines de milliers de $\mathrm{km}^{2}$ (Merlier, 1962). 